\title{
$g$-MODE EXCITATION DURING THE PRE-EXPLOSIVE SIMMERING OF TYPE Ia SUPERNOVAE
}

\author{
AnTHONY L. Piro \\ Theoretical Astrophysics, California Institute of Technology, 1200 E California Blvd., M/C 350-17, \\ Pasadena, CA 91125, USA; piro@ caltech.edu \\ Received 2011 May 3; accepted 2011 July 22; published 2011 August 8
}

\begin{abstract}
Prior to the explosive burning of a white dwarf (WD) that makes a Type Ia supernova (SN Ia), the star "simmers" for $\sim 10^{3} \mathrm{yr}$ in a convecting, carbon-burning region. I estimate the excitation of $g$-modes by convection during this phase and explore their possible effect on the WD. As these modes propagate from the core of the WD toward its surface, their amplitudes grow with decreasing density. Once the modes reach nonlinear amplitudes, they break and deposit their energy into a shell of mass $\sim 10^{-4} M_{\odot}$. This raises the surface temperature by $\approx 4 \times 10^{8} \mathrm{~K}$, which is sufficient to ignite a layer of helium, as is expected to exist for some SN Ia scenarios. This predominantly synthesizes ${ }^{40} \mathrm{Ca}$, but some amount of ${ }^{28} \mathrm{Si}$, ${ }^{32} \mathrm{~S}$, and ${ }^{44} \mathrm{Ti}$ may also be present. These ashes are expanded out with the subsequent explosion up to velocities of $\sim 20,000 \mathrm{~km} \mathrm{~s}^{-1}$, which may explain the high velocity features (HVFs) seen in many SNe Ia. The appearance of HVFs would therefore be a useful discriminant for determining between progenitors, since a flammable helium-rich layer will not be present for accretion from a $\mathrm{C} / \mathrm{O} \mathrm{WD}$ as in a merger scenario. I also discuss the implications of ${ }^{44} \mathrm{Ti}$ production.
\end{abstract}

Key words: convection - stars: oscillations - supernovae: general - white dwarfs

\section{INTRODUCTION}

The use of Type Ia supernovae ( $\mathrm{SNe}$ Ia) as cosmological distance indicators has brought attention to the uncertainties that remain about these events. It is generally agreed that they result from the unstable thermonuclear ignition of a $\mathrm{C} / \mathrm{O}$ white dwarf (WD), but the exact progenitor is still unclear. It may be a single degenerate (WD accreting from an evolved companion; Whelan \& Iben 1973), double degenerate (merging of two WDs; Webbink 1984; Iben \& Tutukov 1984; Paczyński 1985), or combination of both scenarios. Any observational or theoretical clues that could help unravel this mystery are extremely useful.

In cases when the WD first unstably ignites carbon at its center, it subsequently undergoes $\sim 10^{3} \mathrm{yr}$ of convective simmering before the explosive burning wave is born (Woosley et al. 2004; Wunsch \& Woosley 2004). In single degenerate scenarios, central ignition occurs when the accretion proceeds at rates slower than the WD thermal timescale (Hernanz et al. 1988). Central ignition can also occur via focusing of a shock from a surface helium detonation, but this leads to core detonation and not convection (Fink et al. 2010). During the late stages of convection there is considerable luminosity going into convective motions $\left(\sim 10^{45} \mathrm{erg} \mathrm{s}^{-1}\right)$, which is much greater than the Eddington luminosity of a Chandrasekhar WD of $L_{\text {Edd }} \approx 2 \times 10^{38} \mathrm{erg} \mathrm{s}^{-1}$. This energy is expected to be bottled up within the convective region because the thermal conduction timescale is $\sim 10^{6} \mathrm{yr}$, which is much longer than the convective timescale. If just a small fraction of this energy could be transported closer to the WD surface, it might have an effect on the surface structure, and may even have important observable consequences.

In the present work I consider the stochastic excitation of $g$-modes by convection (Goldreich \& Kumar 1990) and how they may affect the WD surface. The presence of such modes, which convert convective energy into traveling waves, was first suggested by Piro \& Chang (2008) and subsequently observed in the simulations of Zingale et al. (2009). In Section 2, I make analytic estimates for the luminosity and total integrated energy in $g$-modes. I show how the modes grow as they propagate toward shallower densities and argue that the mode energy is deposited near the WD surface when the modes break due to reaching nonlinear amplitudes. In Section 3, I study the detailed structure of the heated WD surface layers. The temperature rises sufficiently to ignite a surface helium layer, and the resulting ashes are predominantly composed of ${ }^{40} \mathrm{Ca}$, and perhaps ${ }^{28} \mathrm{Si}$, ${ }^{32} \mathrm{~S}$, and ${ }^{44} \mathrm{Ti}$ will also be present. This may explain the high velocity features (HVFs; Mazzali et al. 2005b) seen in many SNe Ia. In Section 4, I summarize this study and discuss where possible future work is needed.

\section{2. $g$-MODE EXCITATION AND PROPAGATION}

Ignition of ${ }^{12} \mathrm{C}$ occurs when the heating from carbon fusion overpowers neutrino cooling (Nomoto et al. 1984). The central temperature $T_{c}$ then rises and a convective core grows, eventually encompassing $\sim 1 M_{\odot}$ of the WD after $\sim 10^{3} \mathrm{yr}$. For a central density $\rho_{c}$, the energy generation rate from carbon burning is (Woosley et al. 2004)

$$
\epsilon=2.8 \times 10^{13}\left(\frac{T_{c, 8}}{7}\right)^{23}\left(\frac{\rho_{c, 9}}{2}\right)^{3.3} \operatorname{erg~g}^{-1} \mathrm{~s}^{-1},
$$

where $T_{c, 8}=T_{c} / 10^{8} \mathrm{~K}$ and $\rho_{c, 9} \equiv \rho_{c} / 10^{9} \mathrm{~g} \mathrm{~cm}^{-3}$, and equal mass fractions of carbon and oxygen are assumed. The central temperature increases on a heating timescale $t_{h}=$ $\left(d \ln T_{c} / d t\right)^{-1}$, which gets shorter as $T_{c}$ becomes larger, and generally depends on the size of the convecting region (Piro $\&$ Chang 2008). The simmering ends once $t_{h}<t_{c}$, where $t_{c}$ is the eddy overturn timescale. At these late times, individual eddies may experience significant heating during their transit (Garcia-Senz \& Woosley 1995), and there is not sufficient time for the entire convective region to respond to the increasing $T_{c}$. In this case it can be approximated that $t_{h} \approx c_{p} T_{c} / \epsilon$, where $c_{p}$ is the specific heat capacity at constant pressure. The heat capacity in the WD core, including Coulomb corrections, is $c_{p} \approx 1.3 \times 10^{7} \mathrm{erg} \mathrm{g}^{-1} \mathrm{~K}^{-1}$, which is used to find

$$
t_{h} \approx 3 \times 10^{2}\left(\frac{T_{c, 8}}{7}\right)^{-22}\left(\frac{\rho_{c, 9}}{2}\right)^{-3.3} \mathrm{~s} .
$$


Woosley et al. (2004), using the KEPLER code (Weaver et al. 1978), estimate that convection ends when $T_{c} \approx 7.8 \times 10^{8} \mathrm{~K}$ and $\rho_{c} \approx 2.6 \times 10^{9} \mathrm{~g} \mathrm{~cm}^{-3}$. This gives $t_{h} \sim 10 \mathrm{~s}$, roughly the convective overturn timescale as shown below.

Integrating over the burning region of the core, the total luminosity carried by convection is (Woosley et al. 2004)

$$
L_{c} \approx 7 \times 10^{44}\left(\frac{T_{c, 8}}{7}\right)^{23}\left(\frac{\rho_{c, 9}}{2}\right)^{4.3} \mathrm{erg} \mathrm{s}^{-1} .
$$

The convective velocity at the largest scales is $V_{c} \approx$ $\left(L_{c} / 4 \pi r^{2} \rho\right)^{1 / 3}$. What is crucial for driving $g$-modes is the properties near the top of the convective zone, which has density and radius $\rho_{t}$ and $r_{t}$, respectively. The velocity here is

$$
V_{c} \approx 4 \times 10^{6} \rho_{t, 8}^{-1 / 3} r_{t, 8}^{-2 / 3}\left(\frac{T_{c, 8}}{7}\right)^{7.7}\left(\frac{\rho_{c, 9}}{2}\right)^{1.4} \mathrm{~cm} \mathrm{~s}^{-1}
$$

where $\rho_{t, 8}=\rho_{t} / 10^{8} \mathrm{~g} \mathrm{~cm}^{-3}$ and $r_{t, 8}=r_{t} / 10^{8} \mathrm{~cm}$. The spectrum of $g$-modes excited by the convection is peaked at a frequency equal to the eddy turnover frequency $\omega_{c} \approx V_{c} / H_{t}$, where $H_{t}$ is the scale height at the top of the convection. Taking $H_{t} \approx 2 \times 10^{7} \rho_{t, 8}^{1 / 3} g_{10}^{-1} \mathrm{~cm}$, where $g_{10}=g / 10^{10} \mathrm{~cm} \mathrm{~s}^{-2}$, results in

$$
\omega_{c} \approx 0.2 g_{10} \rho_{t, 8}^{-2 / 3} r_{t, 8}^{-2 / 3}\left(\frac{T_{c, 8}}{7}\right)^{7.7}\left(\frac{\rho_{c, 9}}{2}\right)^{1.4} \mathrm{~s}^{-1}
$$

Note the convective timescale is $t_{c} \sim H_{t} / V_{c} \sim 10 \mathrm{~s}$, roughly in agreement with when the convection should end, as discussed above. These waves propagate in the non-convective WD surface layers if their frequency satisfies $\omega_{c}<N$, where $N$ is the Brunt-Väisälä frequency. This is approximated as

$$
N \approx\left(\frac{g}{H} \frac{k_{\mathrm{B}} T_{t}}{Z E_{\mathrm{F}}}\right)^{1 / 2} \approx 0.5 g_{10} T_{t, 8}^{1 / 2} \rho_{8}^{-1 / 3} \mathrm{~s}^{-1}
$$

where $\rho$ (no subscript) is the density at some position near the WD surface, $\rho_{8}=\rho / 10^{8} \mathrm{~g} \mathrm{~cm}^{-3}, k_{\mathrm{B}}$ is Boltzmann's constant, $T_{t}$ is the temperature at the top of the convection, $Z \approx 7$ is the average charge per ion, and $E_{\mathrm{F}}$ is the Fermi energy for a degenerate, relativistic electron gas. Since $\omega_{c}<N$ at the convective boundary, and at shallower depths $N \propto \rho^{-1 / 3}$, the $g$-modes propagate freely toward the surface.

The fraction of $L_{c}$ that can be put into $g$-modes is directly proportional to the Mach number of the convective eddies near the top of the convective zone (Goldreich \& Kumar 1990) ${ }^{1}$. For a sound speed $c_{s}=(4 P / 3 \rho)^{1 / 2}$,

$$
M a=\frac{V_{c}}{c_{s}} \approx 7 \times 10^{-3} \rho_{t, 8}^{-1 / 2} r_{t, 8}^{-2 / 3}\left(\frac{T_{c, 8}}{7}\right)^{7.7}\left(\frac{\rho_{c, 9}}{2}\right)^{1.4}
$$

The $g$-mode luminosity is then

$$
\begin{aligned}
L_{g} \approx & M a L_{c} \approx 5 \times 10^{42} \rho_{t, 8}^{-1 / 2} r_{t, 8}^{-2 / 3}\left(\frac{T_{c, 8}}{7}\right)^{30.7} \\
& \times\left(\frac{\rho_{c, 9}}{2}\right)^{5.7} \operatorname{erg~s}^{-1} .
\end{aligned}
$$

\footnotetext{
1 Some energy is expected to go into $p$-modes as well, but because the efficiency is $\sim M a^{15 / 2}$, this is negligible.
}

Comparing the dependence on $T_{c}$ in Equations (8) and (2) shows that $L_{g} \propto t_{h}^{-1.4}$. The total amount of energy put into $g$-modes up to any given time is therefore

$$
\begin{aligned}
E_{g}= & \int L_{g} d t \approx 2.5 L_{g} t_{h} \approx 4 \times 10^{45} \rho_{t, 8}^{-1 / 2} r_{t, 8}^{-2 / 3}\left(\frac{T_{c, 8}}{7}\right)^{8.7} \\
& \times\left(\frac{\rho_{c, 9}}{2}\right)^{2.4} \mathrm{erg} .
\end{aligned}
$$

Depending the final $T_{c}$ and $\rho_{c}$, about $\sim 10^{46}$ erg can go into $g$-modes.

As the $g$-modes propagate into the non-convective surface layers, they satisfy the dispersion relation

$$
\omega_{c}^{2}=\frac{k_{h}^{2}}{k_{r}^{2}+k_{h}^{2}} N^{2}
$$

and have a group velocity of $V_{g}=\omega_{c} / k_{r}$, where $k_{r}$ and $k_{h}$ are the radial and horizontal wavenumbers, respectively. This relation does not include rotational modifications. The radial wavenumber is unaffected by the Coriolis force if the spin $\Omega$ is small in comparison to the buoyancy (Chapman \& Lindzen 1970; Brekhovskikh \& Goncharov 1994):

$$
\Omega \lesssim N^{2} H / \omega r \sim 0.4 \mathrm{~s}^{-1}
$$

In this limit the mode equations can be simplified using the "traditional approximation" to separate vertical and horizontal parts. Although $k_{r}$ remains the same, $k_{h}$ can depend on the angle with respect to the rotation axis, since the Coriolis force pushes the modes to be more concentrated near the equator (see the angular eigenfunctions plotted in Piro \& Bildsten 2004). The spin of accreting WDs may indeed exceed that given by Equation (11), but rotational modifications are ignored to simplify the current study.

The total luminosity in $g$-modes is related to the Lagrangian displacement $\xi$ via (Mihalas \& Toomre 1981)

$$
L_{g} \approx 8 \pi r^{2} \rho(\omega \xi)^{2} V_{g}
$$

From incompressibility, the components of $\xi^{2}=\xi_{r}^{2}+\xi_{h}^{2}$ are related by $\left(\xi_{r} / \xi_{h}\right)^{2} \approx\left(k_{h} / k_{r}\right)^{2}$. The linearity of the modes is best represented by the dimensionless quantity $k_{r} \xi_{r}$, since both density inversion instabilities and Kelvin-Helmholtz instability set in when $k_{r} \xi_{r} \sim O(1)$ (Mihalas \& Toomre 1981). For example, it is simple to show that $k_{r} \xi_{r} \sim k_{r} V_{h} / N$, where $V_{h}=\omega \xi_{h}$ is the horizontal velocity, is just the Richardson number determining shear stability. Using the above relations, in the limit $\omega \lesssim N$, the amplitude simplifies to

$$
k_{r} \xi_{r} \approx\left(\frac{L_{g} N}{8 \pi r^{2} \rho}\right)^{1 / 2} \frac{k_{h}^{3 / 2}}{\omega^{2}}
$$

and taking $k_{h} \approx 1 / H_{t}$, I evaluate this as a function of $\rho$,

$$
k_{r} \xi_{r} \approx 8 \times 10^{-2} \rho_{t, 8}^{7 / 12} r_{t, 8}^{-1} T_{t, 8}^{1 / 4} \rho_{7.7}^{-2 / 3}
$$

As the mode propagates toward the surface $k_{r} \xi_{r} \propto \rho^{-1 / 2} N^{1 / 2} \propto$ $\rho^{-2 / 3}$, so this dimensionless amplitude grows. This quantity is independent of the strength of convection, as apparent from the lack of a dependence on $T_{c}$ or $\rho_{c}$. This is because the amplitude is $k_{r} \xi_{r} \propto L_{g}^{1 / 2} \omega^{-2}$. As the driving becomes more vigorous, 
the $g$-mode luminosity increases, but so does the frequency, and the two effects exactly balance ${ }^{2}$.

Near the surface the thermal conduction timescale becomes shorter. The thermal conductivity from electron-ion scattering is (Yakovlev \& Urpin 1980)

$$
K=\frac{\pi^{2} k_{\mathrm{B}}^{2} T n_{e}}{3 m_{*} \nu},
$$

where $m_{*} \approx E_{\mathrm{F}} / c^{2}, n_{e}$ is the number density of electrons, and $v=4 m_{*} Z e^{4} \Lambda / 3 \pi \hbar^{3}$ is the electron-ion collision frequency with $\Lambda \approx 1$ is a weakly dependent function of density in the ocean and $\hbar$ is Planck's constant. For a length scale $\lambda$, the local thermal time is $t_{\mathrm{th}} \sim \rho c_{p} \lambda^{2} / K$, giving

$$
t_{\text {th }} \approx 6 \times 10^{5} \rho_{8}^{2 / 3} T_{t, 8}^{-1}\left(\frac{\lambda}{2 \times 10^{7} \mathrm{~cm}}\right)^{2} \mathrm{yr},
$$

where $\lambda$ has been scaled to the typical horizontal wavelength of the $g$-modes. This is much longer than the timescale for a mode to travel a scale height $t_{g} \approx H / V_{g} \approx\left(H / H_{t}\right) / \omega_{c} \sim$ tens of seconds, so conduction does not damp the modes. Since breaking occurs before damping, setting $k_{r} \xi_{r} \approx 1$, I find

$$
\rho_{b} \approx 2 \times 10^{6} \rho_{t, 8}^{7 / 8} T_{t, 8}^{3 / 8} \mathrm{~g} \mathrm{~cm}^{-3}
$$

for the mode breaking density.

\section{SURFACE HEATING}

For the breaking depth found in Equation (17), the corresponding mass of material is $M_{b} \sim 4 \pi r^{2} \rho_{b} H\left(\rho_{b}\right) \sim 10^{-4} M_{\odot}$. If an energy $E_{g}$ is put into a shell with mass $M_{b}$, it does not eject the material, since the binding energy for a Chandrasekhar WD is much greater at $G M M_{b} / R \sim 10^{47} \mathrm{erg}$. Instead the energy input heats the surface, which I now explore.

A simple estimate for the change of temperature is $\Delta T \sim$ $E_{g} / c_{p} M_{b} \sim$ few $\times 10^{8} \mathrm{~K}$, but this does not take into account how the heating is distributed. Heating at a depth $\rho_{b}$ acts like a hot plate, which still cannot be carried by conduction due to the long thermal timescale (see Equation (16), evaluated at $\rho_{b}$ ). Instead, a secondary convective zone grows, which distributes the heat over a wider region.

To better understand the extent and peak temperature of this secondary convection zone, I build a series hydrostatic surface models where the convection zone is treated as an adiabat. The initial model is assumed isothermal with a temperature of $T_{i}=6 \times 10^{7} \mathrm{~K}$, similar to the surface temperature in accreting WD models (Yoon \& Langer 2004). The convection extends from a bottom mass coordinate $M_{1}$ set by the $g$-mode breaking depth up to top mass coordinate $M_{2}$ set by the total energy injected by the $g$-modes:

$$
E_{g}=\int_{M_{1}}^{M_{2}} c_{p}\left[T\left(M_{r}\right)-T_{i}\right] d M_{r}
$$

where $T\left(M_{r}\right)$ is the temperature profile. This integral is fairly insensitive to the assumption of $T_{i}$, since at late times the convection has much more energy than the initial temperature profile. Even though $M_{1}$ is set fixed by the mode breaking depth, the density at the base of the convective zone decreases because of the increasing temperature at fixed pressure.

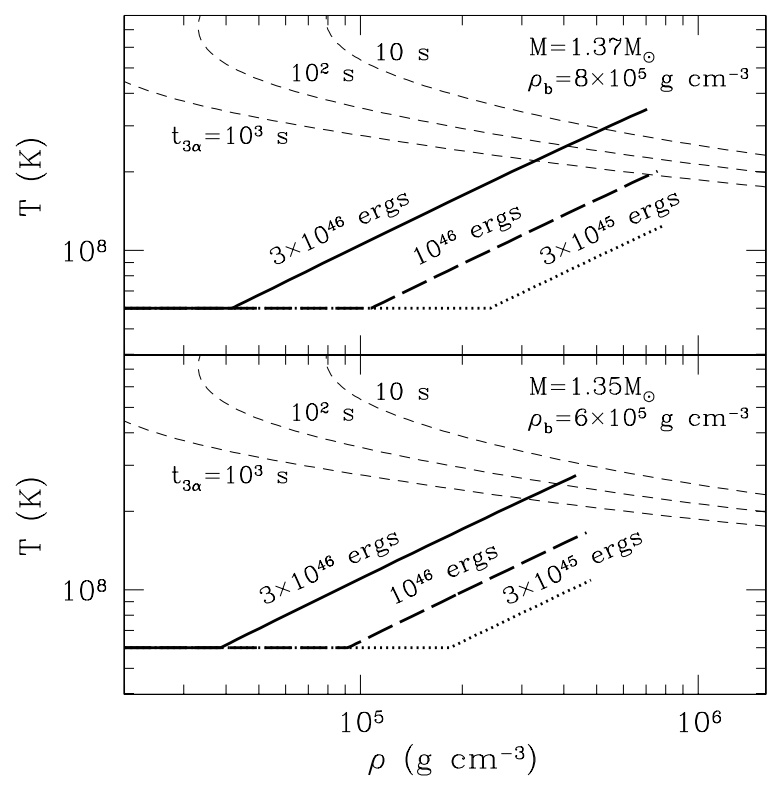

Figure 1. Example temperature profiles for the surface convection zone caused by energy deposited from $g$-modes. In the top panel I use $M=1.37 M_{\odot}$, $R=1.6 \times 10^{8} \mathrm{~cm}$, and $\rho_{b}=8 \times 10^{5} \mathrm{~g} \mathrm{~cm}^{-3}$, and in the bottom panel $M=1.35 M_{\odot}, R=2.1 \times 10^{8} \mathrm{~cm}$, and $\rho_{b}=6 \times 10^{5} \mathrm{~g} \mathrm{~cm}^{-3}$. The thick lines show convective profiles for different amounts of total internal energy, as labeled. The thin, short-dashed lines are curves of constant $t_{3 \alpha}$, demonstrating when helium burning begins.

In Figure 1, I plot example thermal profiles that would be present from energy deposited by $g$-modes. In the top panel I use $M=1.37 M_{\odot}, R=1.6 \times 10^{8} \mathrm{~cm}$, and $\rho_{b}=8 \times 10^{5} \mathrm{~g} \mathrm{~cm}^{-3}$, and in the bottom panel $M=1.35 M_{\odot}, R=2.1 \times 10^{8} \mathrm{~cm}$, and $\rho_{b}=6 \times 10^{5} \mathrm{~g} \mathrm{~cm}^{-3}$. The central densities of the two models are $6.1 \times 10^{9} \mathrm{~g} \mathrm{~cm}^{-3}$ and $2.0 \times 10^{9} \mathrm{~g} \mathrm{~cm}^{-3}$, respectively. The difference in breaking depths was estimated from coreconvective models (as calculated in Piro \& Bildsten 2008; Piro 2008). Each thick line shows the thermal profile for a labeled amount of $g$-mode energy $E_{g}$, solved using Equation (18). The more massive WD is plotted with a larger $E_{g}$ due to its higher central density (Equation (9)). The base temperature rises by $\Delta T \approx 4 \times 10^{8} \mathrm{~K}$ for the top panel and by $\approx 3 \times 10^{8} \mathrm{~K}$ for the bottom panel, but for a given $E_{g}, \Delta T$ is roughly the same. These detailed profiles also provide an estimate of the position of the top of the convection zone, which is near $\sim 10^{4}-10^{5} \mathrm{~g} \mathrm{~cm}^{-3}$. If the thermal time here is sufficiently short, heat leaves the top of the convection and stunts its growth, as is found for Type I X-ray bursts (Weinberg et al. 2006). Since the thermal time $t_{\mathrm{th}} \sim 10 \mathrm{yr}$ (Equation (16)) is much longer than $t_{h}$, conduction does not truncate the secondary convective zone.

Besides changing the entropy and thermal profile, the heating may have an additional effect if the surface layers are rich in helium. A thin layer of helium is expected in many single degenerate scenarios that require accretion to grow to a Chandrasekhar mass. For an energy generation rate from triple$\alpha$ reactions of $\epsilon_{3 \alpha}$, the timescale for increasing the temperature is $t_{3 \alpha} \approx c_{p} T / \epsilon_{3 \alpha}$. Figure 1 plots $t_{3 \alpha}=10,10^{2}$, and $10^{3} \mathrm{~s}$ (thin, short-dashed lines), using the reaction rates from Fushiki \& Lamb (1987). Comparison of these curves with the convective profiles demonstrates that triple- $\alpha$ reactions can begin burning

\footnotetext{
2 In detail, as the convection proceeds $\rho_{t}$ decreases, but this is a small correction with respect to the simplifying assumptions I am making here.
} 
the layer. Helium burning favors deeper mode breaking and more massive WDs, since a higher density leads to a shorter $t_{3 \alpha}$. A more detailed study is required to assess the full range of WD masses and breaking depths needed for helium ignition, which is outside the scope of this work.

The low shell mass is below what is needed for dynamical burning (Shen \& Bildsten 2009), so a detonation is not expected. Nevertheless, energy from helium burning provides $\approx 5.8 \times$ $10^{17} \mathrm{erg} \mathrm{g}^{-1}$, similar to the binding energy of a Chandrasekhar WD $\left(\sim 10^{18} \mathrm{erg} \mathrm{g}^{-1}\right)$, so it is possible that some material is expelled. Typical breaking depths correspond to a base pressure of $\approx(3-5) \times 10^{22} \mathrm{erg} \mathrm{cm}^{-3}$. The burning of helium at constant pressure was explored by Hashimoto et al. (1983), the products of which are summarized in their Figure 10. For this pressure range, the predominant element is ${ }^{40} \mathrm{Ca}$, but ${ }^{44} \mathrm{Ti}$ may also be present. As the burning proceeds out to lower densities, other $\alpha$-elements such as ${ }^{28} \mathrm{Si},{ }^{32} \mathrm{~S}$, and ${ }^{36} \mathrm{Ar}$ would also be synthesized.

The composition and position of these elements are similar to the HVFs seen in many (or perhaps most) SNe Ia (Mazzali et al. 2005b). HVFs most prominently show ${ }^{40} \mathrm{Ca}$, although ${ }^{28} \mathrm{Si}$ is sometimes present. Currently explanations for these features include circumstellar interactions (Gerardy et al. 2004), threedimensional density or composition enhancements (Mazzali et al. 2005a), or ashes on the surface from a gravitationally confined explosion (Kasen \& Plewa 2005). Modeling of the early time light curve, including the HVFs, was done by Tanaka et al. (2008). If HVFs are due to surface burning from $g$-mode energy input, their presence would be an indication that helium accretion (or hydrogen accretion which then burns to form helium) is responsible for these $\mathrm{SNe}$ Ia, which is expected for a single degenerate progenitor or a degenerate helium donor (like for AM CVn stars).

\section{CONCLUSION AND DISCUSSION}

I considered the driving of $g$-modes via convection during the simmering stage prior to SNe Ia. I found that the $g$-modes are driven with a luminosity $L_{g} \gtrsim 10^{42} \mathrm{erg} \mathrm{s}^{-1}$, injecting $E_{g} \sim 10^{46} \mathrm{erg}$ at a density of $\rho_{b} \sim 10^{6} \mathrm{~g} \mathrm{~cm}^{-3}$ where the modes break. I considered the effect of this energy in generating a secondary, surface convective region and showed it reaches base temperatures of $\approx 4 \times 10^{8} \mathrm{~K}$, sufficient to ignite helium. The ashes expected from this burning are similar to the elements observed in HVFs, which may mean that HVFs indicate a progenitor channel different from the merger of two C/O WDs since these would not have helium present. Future studies should be done to assess how ubiquitous HVFs really are among the variety of types of SNe Ia.

This study motivates further, more detailed investigations. The mode excitation estimates employed here make use of studies done in the context of the Sun (Goldreich \& Kumar 1990) and may need modification for a degenerate equation of state. In addition, rotation can strongly influence the morphology of WD convection (Kuhlen et al. 2006), which would in turn alter the mode wavelengths and spectrum (also see the discussion of rotational modifications in Section 2). Rotation breaks the spherical symmetry of the WD and would imprint asymmetries into the flux of $g$-modes. If magnetic fields are driven toward equipartition by a convective dynamo (Piro \& Chang 2008), this may also affect the modes.

A study of thin helium-shell burning on massive WDs would provide detailed predictions for the burning products and determine what fraction of material can be expelled. The production of ${ }^{44} \mathrm{Ti}$ is especially interesting for testing this model and investigating SNe Ia progenitors. Too much ${ }^{44} \mathrm{Ti}$ near the surface causes early time absorption and reddening, inconsistent with usual SN Ia light curves (Kromer et al. 2010). Typically $\sim 10^{-5} M_{\odot}$ of ${ }^{44} \mathrm{Ti}$ is produced by surface burning, although this varies strongly as a function of $\rho_{b}$. Borkowski et al. (2010) find (1-7) $\times 10^{-5} M_{\odot}$ of ${ }^{44} \mathrm{Ti}$ in the remnant $\mathrm{G} 1.9+0.3$ from the decay product ${ }^{44} \mathrm{Sc}$. The ${ }^{44} \mathrm{Sc}$ is most abundant in the remnant's north rim, which is surprising if the ${ }^{44} \mathrm{Ti}$ is expected to be synthesized in the neighborhood of more centrally located Fe-peak elements (Iwamoto et al. 1999). Further studies of the distribution of ${ }^{44} \mathrm{Sc}$ would help determine whether surface helium burning is a viable alternative. Detections of the $68 \mathrm{keV}$ decay line from ${ }^{44} \mathrm{Ti}$ in SNe Ia by future satellites like NuSTAR (Harrison et al. 2010) may also provide an important constraint. A mass $M_{\mathrm{Ti}}$ of ${ }^{44} \mathrm{Ti}$ synthesized implies a count rate at time $t$ of

$$
r_{68 \mathrm{keV}} \approx 10^{-3} A_{100} d_{10}^{-2}\left(\frac{M_{\mathrm{Ti}}}{10^{-5} M_{\odot}}\right) e^{-t / t_{\mathrm{Ti}}} \text { counts s}^{-1}
$$

where $A=100 A_{100} \mathrm{~cm}^{2}$ is the effective area of detection, $d=10 d_{10} \mathrm{kpc}$ is the distance, and $t_{\mathrm{Ti}}=85 \mathrm{yr}$ is the ${ }^{44} \mathrm{Ti}$ decay timescale.

I thank Philip Chang for some of the original conversations that inspired this research, and Lars Bildsten, Peter Goldreich, Fiona Harrison, Paolo Mazzali, Christian Ott, Eliot Quataert, Nevin Weinberg, and Stan Woosley for helpful feedback. This work was supported through NASA ATP grant NNX07AH06G, NSF grant AST-0855535, and by the Sherman Fairchild Foundation.

\section{REFERENCES}

Borkowski, K. J., Reynolds, S. P., Green, D. A., et al. 2010, ApJ, 724, L161

Brekhovskikh, L. M., \& Goncharov, V. 1994, Mechanics of Continua and Wave Dynamics (Berlin: Springer)

Chapman, S., \& Lindzen, R. S. 1970, Atmospheric Tides (Dordrecht: Reidel)

Fink, M., Röpke, F. K., Hillebrandt, W., et al. 2010, A\&A, 514, A53

Fushiki, I., \& Lamb, D. Q. 1987, ApJ, 317, 368

Garcia-Senz, D., \& Woosley, S. E. 1995, ApJ, 454, 895

Gerardy, C. L., Höflich, P., Fesen, R. A., et al. 2004, ApJ, 607, 391

Goldreich, P., \& Kumar, P. 1990, ApJ, 363, 694

Harrison, F. A., Boggs, S., Christensen, F., et al. 2010, Proc. SPIE, 7732 $77320 \mathrm{~S}$

Hashimoto, M.-A., Hanawa, T., \& Sugimoto, D. 1983, PASJ, 35, 1

Hernanz, M., Isern, J., Canal, R., et al. 1988, ApJ, 324, 331

Iben, I. J., \& Tutukov, A. V. 1984, ApJS, 54, 335

Iwamoto, K., Brachwitz, F., Nomoto, K., et al. 1999, ApJS, 125, 439

Kasen, D., \& Plewa, T. 2005, ApJ, 622, L41

Kromer, M., Sim, S. A., Fink, M., et al. 2010, ApJ, 719, 1067

Kuhlen, M., Woosley, S. E., \& Glatzmaier, G. A. 2006, ApJ, 640, 407

Mazzali, P. A., Benetti, S., Stehle, M., et al. 2005a, MNRAS, 357, 200

Mazzali, P. A., Benetti, S., Altavilla, G., et al. 2005b, ApJ, 623, L37

Mihalas, B. W., \& Toomre, J. 1981, ApJ, 249, 349

Nomoto, K., Thielemann, F.-K., \& Yokoi, K. 1984, ApJ, 286, 644

Paczyński, B. 1985, in Cataclysmic Variables and Low-Mass X-Ray Binaries, ed. D. Q. Lamb \& J. Patterson (Dordrecht: Reidel), 1

Piro, A. L. 2008, ApJ, 679, 616

Piro, A. L., \& Bildsten, L. 2004, ApJ, 603, 252

Piro, A. L., \& Bildsten, L. 2008, ApJ, 673, 1009

Piro, A. L., \& Chang, P. 2008, ApJ, 678, 1158

Shen, K. J., \& Bildsten, L. 2009, ApJ, 699, 1365

Tanaka, M., Mazzali, P. A., Benetti, S., et al. 2008, ApJ, 677, 448

Weaver, T. A., Zimmerman, G. B., \& Woosley, S. E. 1978, ApJ, 225, 1021 
Webbink, R. F. 1984, ApJ, 277, 355

Weinberg, N. N., Bildsten, L., \& Schatz, H. 2006, ApJ, 639, 1018

Whelan, J., \& Iben, I., Jr. 1973, ApJ, 186, 1007

Woosley, S. E., Wunsch, S., \& Kuhlen, M. 2004, ApJ, 607, 921
Wunsch, S., \& Woosley, S. E. 2004, ApJ, 616, 1102

Yakovlev, D. G., \& Urpin, V. A. 1980, SvA, 24, 303

Yoon, S.-C., \& Langer, N. 2004, A\&A, 419, 645

Zingale, M., Almgren, A. S., Bell, J. B., Nonaka, A., \& Woosley, S. E. 2009, ApJ, 704,196 